\title{
Artificial Intelligence for Advanced Building Control: Energy and GHG Savings from a Case Study ${ }^{\dagger}$
}

\author{
Nunzio Cotrufo * and Etienne Saloux \\ CanmetENERGY, Natural Resources Canada, 1615 Blvd. Lionel-Boulet, Varennes, QC J3X 1P7, Canada \\ * Correspondence nunzio.cotrufo@canada.ca \\ + Presented at the Symposium on Energy Efficiency in Buildings and Industry, Sherbrooke, QC, Canada, \\ 28 May 2019.
}

Published: 28 August 2019

\begin{abstract}
Model-based Predictive Control (MPC) is a promising advanced control strategy for the improvement of building operation. MPC uses a model of the building along with weather forecasts to optimize control strategies, such as indoor air temperature set-points, thermal storage charging and discharging cycles, etc. An obstacle to the adoption of MPC is the modelling step: developing a dedicated control-oriented model is a time-consuming process, requiring technical expertise and a large amount of information about the building and its operation. To overcome these issues, this paper proposes a new approach for the development of MPC strategies based on Artificial Intelligence (AI) techniques, aiming to map correlations among commonly available operation variables and to develop models suitable for predictive control. The proposed approach was applied in an institutional building in Varennes, QC, with the aim of reducing the natural gas consumption during the heating season. Early results show a remarkable effectiveness of the proposed approach, with a reduction of natural gas and building heating consumption of $23.9 \%$ and $6.3 \%$, respectively.
\end{abstract}

Keywords: advanced control; Artificial Intelligence; model-based predictive control; energy efficiency

\section{Introduction}

Buildings often perform below their energy efficiency potential; it has been estimated that building operation improvements could lead to energy savings of the order of 30\% [1]. Large opportunities to reduce building operational costs and resources deployment may be reached through advanced control [2]. For instance, Model-based Predictive Control (MPC) is an advanced control strategy, which enables energy savings and larger use of renewable sources when compared to traditional rule-based control approaches. MPC uses a model along with weather forecasts to predict the building thermal and energy behavior for the next hours. Building control strategies (i.e., set-point profiles, energy storage charge and discharge cycles, etc.) are then optimized in order to minimize a cost function (i.e., total energy consumption, peak demand, utility bill, etc.). A review of control systems for building applications performed by Afram and Janabi-Sharifi [3] indicates that MPC generally performed better than most of the other control strategies. According to Killian and Kozek [4], the main obstacle to the widespread adoption of MPC is the development of effective controloriented models. A dedicated control-oriented model needs to be developed for each building, which requires time, expertise, and often unavailable information. The authors of this paper aimed to leverage Artificial Intelligence (AI) techniques to develop an MPC strategy that requires reduced time and expertise while using a limited amount of commonly available information from the Building Automation System (BAS). Although AI emerged in the last decade as a powerful tool for a wide range of applications (e.g., medical diagnosis, genetics, transportation, etc.), its application for building control is still in its early stages. 
This paper proposes a new AI-based approach for the development of MPC in buildings. An institutional building was considered as case study with the objective of reducing the natural gas consumption and the GHG emissions during the heating season. Results from the implementation of the developed MPC in the building case study are presented and discussed.

\section{Case Study}

In this study, the CanmetENERGY Centre, located in Varennes, QC, was considered as case study. The building is a $3770 \mathrm{~m}^{2}$ single storey building hosting roughly 120 workstations and 10 meeting rooms. Heating is provided by a $170 \mathrm{~kW}$ electric boiler and two $450 \mathrm{~kW}$ natural gas boilers. When heating is needed, the electric boiler is always started first. The natural gas boilers are turned on when the electric boiler cannot meet the heating demand by itself. This is a common configuration for institutional and commercial buildings in Quebec that underwent a retrofit of the heating system. Part of the monthly electric bill is due to the peak electric demand, as the electric energy utility charges per $\mathrm{kW}$ of monthly peak demand. In order to reduce peak demand, the total electrical power supplied to the building, which corresponds to the sum of the electric boiler power and the building electrical baseload (e.g., workstations, lighting, appliances, experimental test benches, etc.), is limited to $230 \mathrm{~kW}$. Since the electric baseload has the priority, the electric boiler power input is kept within the margin between the $230 \mathrm{~kW}$ total power limit and the baseload power. For instance, at night during week days in January, the value of the baseload is about $80 \mathrm{~kW}$. Thus, the electric boiler power input cannot exceed $150 \mathrm{~kW}$ (i.e., $230 \mathrm{~kW}-80 \mathrm{~kW}$ ). Figure 1 shows the profiles of the building electric demand, the electric boiler power input, and the natural gas boiler power input for a few days in January 2018.

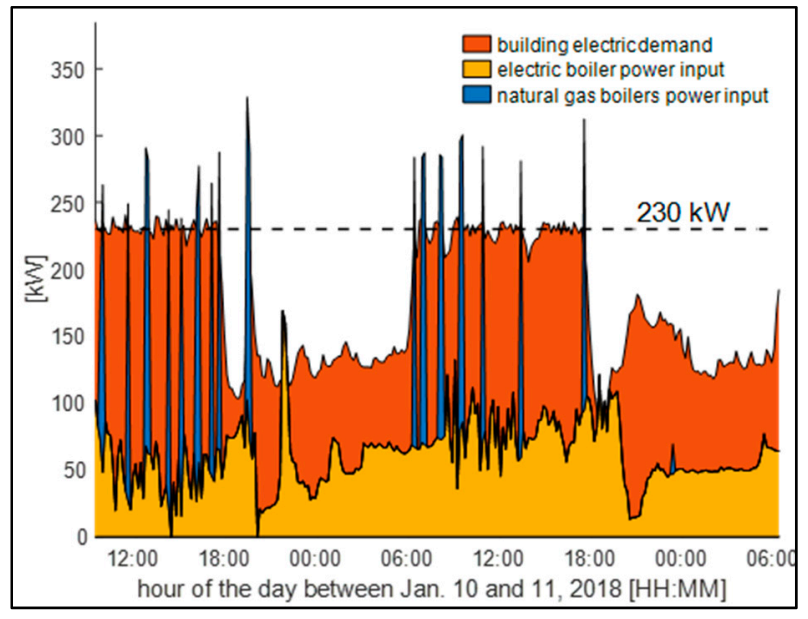

Figure 1. Building case study power profiles during few days in January 2018.

The CanmetENERGY Centre uses a BAS that collects measurements (e.g., temperature, power inputs, etc.) at 10-min intervals. From 2015 to 2018, during winter, the indoor environment was controlled with a night set-back set-point: during the day (7:00-17:30) the indoor temperature was set at $23.0^{\circ} \mathrm{C}$; at night the set-point dropped to $19.6{ }^{\circ} \mathrm{C}$. If the outdoor temperature fell below $-5^{\circ} \mathrm{C}$, the night set-back was raised up to $21.0^{\circ} \mathrm{C}$. This control strategy is here referred to as Business As Usual (BAU). In the BAU strategy, the sudden variation of the set-point profile in the morning provokes a peak in the building heating demand. This peak cannot usually be fulfilled by the electric boiler alone, and the gas boilers are required.

\section{Methodology}

In order to reduce the natural gas consumption, several setpoint profiles with different slopes (i.e., ramps) were considered during nighttime (between 17:30 and 7:00) while the same constant value was considered during daytime $\left(23^{\circ} \mathrm{C}\right.$ between $7: 00$ and 17:30). Figure 2 shows some of the set- 
point profiles considered in this study. The solid line represents the BAU control strategy. The proposed MPC routine implemented in the building consists of the following steps:

1. 24 hours of weather forecasts are retrieved at 16:00;

2. The building model and the weather forecasts are used to assess the building heating demand and the natural gas consumption for each of the considered set-point profiles;

3. The set-point profile leading to the lowest consumption of natural gas (optimal control) is identified;

4. Starting at 18:00, the building is operated under the identified optimal control during the next 24 hours.

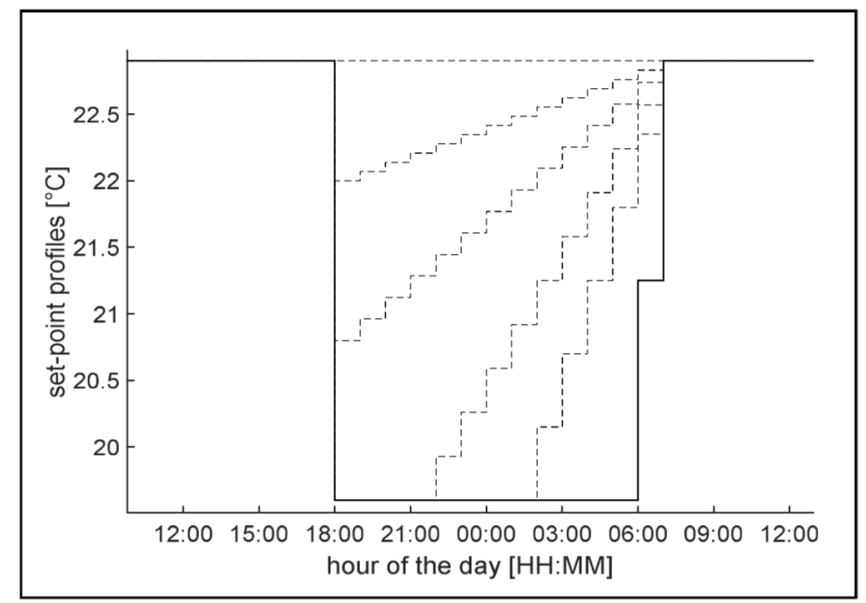

Figure 2. Examples of the considered set-point profiles for reducing the natural gas consumption.

\section{Modelling Approach}

Model architecture: a three-model architecture has been used to model the building response with a one-hour time step (Figure 3).

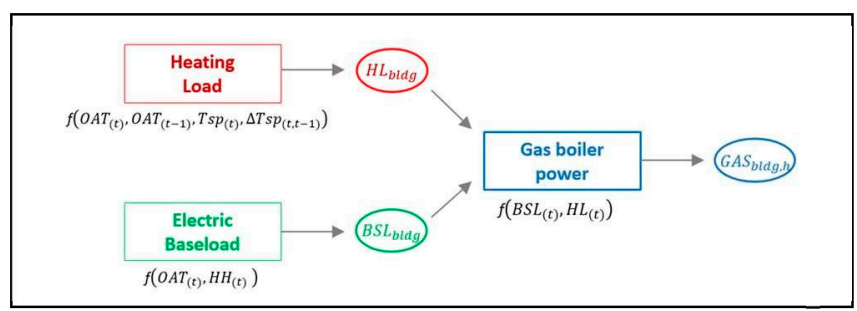

Figure 3. Schematic of the proposed model architecture.

- The first model (red in Figure 3) predicts the building heating load at time $t$ using the outdoor air temperature at time $t$ and time $t-1$, the indoor air set-point $\left(T_{\text {sp }}\right)$ at time $t$, and the set-point variation between time $t$ and $t-1\left(\Delta \mathrm{T}_{\mathrm{sp}}\right)$.

- The second model (green in Figure 3) predicts the building electric baseload at time $t$ using the outdoor air temperature (OAT), and the hour of the day at time $t$.

- Finally, the third model (grey in Figure 3) predicts the gas boilers power input at time $t$ as function of both the building heating demand and the electric baseload.

Modelling techniques: five AI techniques were considered for the development of the threemodel architecture: i) Artificial Neural Network (ANN); ii) Gaussian Process Regression (GPR); iii) Support Vector Machine (SVM); iv) Decision Tree (DT); and v) Random Forest (RF). The accuracy of each technique in predicting the building load was evaluated using statistical indices, and the most effective technique was selected. Measurements from the 2017-2018 winter season were used to train and validate the models. The dataset contained hourly measurements from 83 working days. It was 
randomly divided into two sub-datasets: $50 \%$ of the dataset was kept for model training, and the rest for model validation.

Selection of inputs: AI techniques can be used for modelling building loads in an effective manner, while providing the advantages of requiring less time, effort, information and levels of expertise. However, attention must be paid to the selection of appropriate model inputs. There is a risk of mapping correlations incorrectly. For instance, the building electric baseload and the BAU temperature set-point profile have similar shapes. Therefore, one could incorrectly conclude that the baseload varies with the set-point. In reality, the setpoint was programmed to follow the building occupancy, while the baseload mainly results from the energy used by the occupants (workstations, lighting, appliances, etc.), which also follows the occupancy. However, the driving variable for the baseload here is the occupancy, not the set-point; varying the set-point should not result in a variation of the baseload. Thus, a good understanding of the data and an appropriate selection of model inputs remain essential for the proper development of AI-based control-oriented models.

MPC implementation: the proposed MPC strategy has been implemented in the 2018-2019 winter season and results are presented for the period 14 January-22 February 2019. For every workday, the day before at 16:00, the weather forecasts are automatically retrieved using CanMETEO $^{\circledR}$ [5], a publicly available free-of-charge software tool developed at CanmetENERGY [6]. A Matlab script [7] implements the MPC routine: the weather forecasts are used along with the building model to identify the optimal control set-point profile for the next 24 hours. Finally, the optimal control profile is automatically sent to the BAS and used to operate the building from 18:00 to $17: 59$ of the following day.

Assessment of energy savings: The assessment of energy savings due to MPC implementation is performed by comparing the measured energy consumption under MPC to the expected energy consumption under BAU control. For this purpose, two "energy signature" (benchmark) models of the building were developed. These models predict the building daily average heating load and gas power input under BAU control as function of the outdoor air daily average temperature and the day of the week. Historical data from winters 2015-2018 were used to develop two GPR-based energy signature models. The role of the energy signature models is to provide a benchmark of the building energy consumption, in order to assess the performance of new control strategies [8]. The benchmark models use outdoor air temperature and the day of the week to predict how the BAU strategy would perform.

\section{Results}

This section presents the results of the three-model structure development and the MPC implementation.

Accuracy of the three-model structure: The accuracy of the five considered AI techniques was evaluated by comparing the predictions of the models with the collected measurements. Accuracy was evaluated in terms of Root Mean Square Error (RMSE). Table 1 shows results for the building heating load (red in Figure 3) and the natural gas consumption (blue in Figure 3) for both training and validation periods. RMSE are similar for all techniques; GPR-based models were selected.

Table 1. RMSE of the five AI techniques for building heating load and natural gas consumption predictions.

\begin{tabular}{ccccc}
\hline AI TechniqueHeating Load $(\mathbf{k W})$ & TrainValidNatural & Gas $(\mathbf{k W})$ & TrainValid \\
\hline ANN & 30.1 & 32.6 & 10.1 & 10.4 \\
GPR & 24.3 & 32.1 & 3.3 & 11.2 \\
SVM & 30.2 & 33.9 & 10.2 & 11.7 \\
DT & 27.6 & 32.7 & 8.3 & 12.2 \\
RF & 22.3 & 32.7 & 8.5 & 12.1 \\
\hline
\end{tabular}


MPC results: Early results of the MPC are given in Figure 4 and Figure 5 for the building heating demand and for the natural gas boiler consumption, respectively. In Figures 4 and 5, the grey dots represent the historical data (2015-2018) used to develop the energy signature models (the grey curves. The green dots represent the daily average power values measured during MPC implementation. In these figures, most of green points are below the energy signature: in other words, the MPC outperforms the BAU strategy. Savings are summarized in Table 2. The measured natural gas consumption (33.7 MWh) was $23.9 \%$ lower than that expected under BAU control (44.3 MWh). This roughly corresponds to a reduction of GHG emissions of $1.9 \mathrm{t}$ of $\mathrm{CO}_{2} \mathrm{eq}$ (23.1\% reduction). Moreover, the building heating demand observed under MPC $(92.0 \mathrm{MWh})$ is $6.3 \%$ lower than what would be expected under the BAU scenario ( $98.3 \mathrm{MWh}$ ). So far, the proposed MPC has proved to be effective in reducing the natural gas consumption and the associated GHG emissions.

It is worth highlighting that the daily natural gas consumption was used as the cost function in this study, and no constraints were applied to the building heating demand. Therefore, the MPC routine uses the daily gas consumption as the only criterion to select the optimal control strategy. Under these conditions, it is possible for the daily building heating demand to occasionally increase. In order to guarantee the MPC cost effectiveness, additional constraints must be included in the MPC routine (e.g., the heating demand or the energy bill).

Discussion: The selection of the optimal control strategies by the MPC routine along the implementation period was finally analyzed, and a trend was highlighted: the colder the outdoor air temperature, the higher the slope of the optimal set-point profile, and thus the faster the transition from the night setback to the daytime temperature value $\left(23^{\circ} \mathrm{C}\right)$. On the other hand, the warmer the outdoor air temperature, the "flatter" the optimal set-point profile. This trend can be explained by analyzing the consumption of natural gas at night. When the outdoor air temperature is very low, burning natural gas is required at night; it is thus beneficial to maintain the lower night set-back value longer to avoid excessive consumption. The sudden expenditure of natural gas consumption resulting from the abrupt set-point variation (the morning peak load) would still be less than the gas savings during the night.

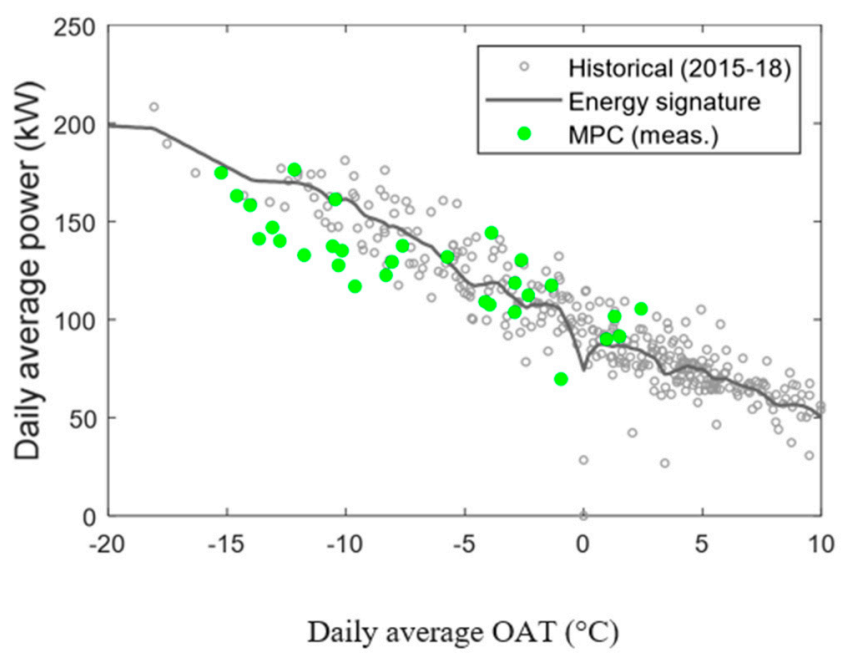

Figure 4. Daily average heating power demand: early results of the MPC implementation (winter 2019). 


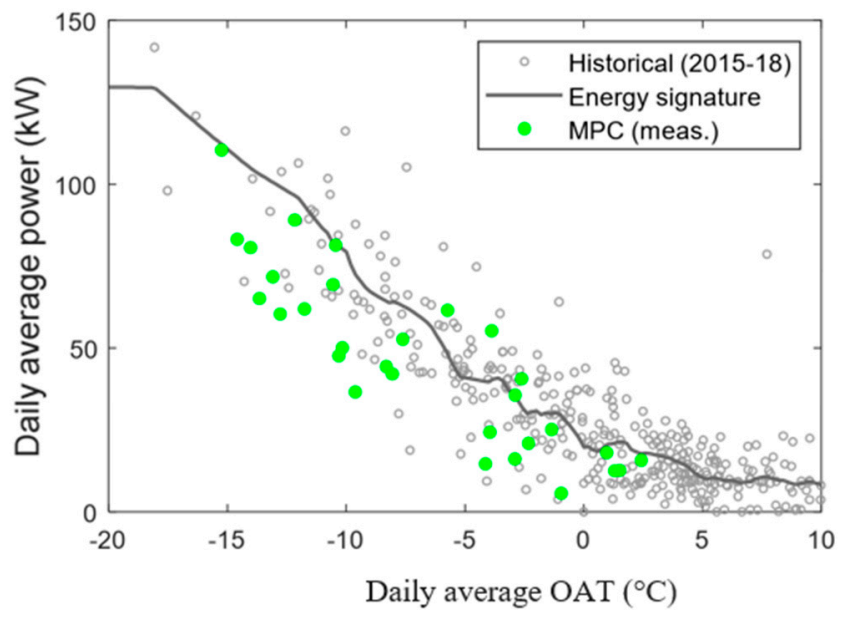

Figure 5. Daily average natural gas power demand: early results of the MPC implementation (winter 2019).

Table 2. Savings obtained from MPC implementation vs BAU operation (early results, winter 2019).

\begin{tabular}{cccc}
\hline Variable & BAU & MPC & Savings \\
\hline Heating demand & $98.3 \mathrm{MWh}$ & $92.0 \mathrm{MWh}$ & $6.3 \%$ \\
Natural gas & $44.3 \mathrm{MWh}$ & $33.7 \mathrm{MWh}$ & $23.9 \%$ \\
GHG emissions & $8.1 \mathrm{t} \mathrm{CO}_{2}$ & $6.2 \mathrm{t} \mathrm{CO}_{2}$ & $23.1 \%$ \\
\hline
\end{tabular}

\section{Conclusions}

A novel approach for the development and implementation of MPC in buildings was presented. AI techniques were used to reduce the time and expertise required for the development stage of the control-oriented model. Furthermore, only measurements commonly available from a typical BAS were needed to train and validate the AI-based three-model structure. The proposed strategy was implemented in an institutional building in Varennes, QC, from 14 January to 22 February 2019. Results revealed that MPC achieved a $23.9 \%$ reduction of natural gas consumption $\left(1.9 \mathrm{t}\right.$ of $\mathrm{CO}_{2}$ equivalent) and a $6.3 \%$ reduction in building heating demand. AI-based MPC can effectively support the widespread adoption of advanced control strategies in buildings. Future work might include the uncertainty analysis of the control-oriented models, and the impact of this uncertainty on the MPC results. The uncertainty associated to the energy signature models should be investigated as well. Finally, the MPC routine could be used to generate a large amount of simulations in order to extract advanced control rules. These rules may be used, along with weather forecasts, to control the building without performing daily MPC optimization routines.

Author Contributions: N.C. and E.S. conceived and designed the approach presented in this paper; N.C. and E.S. analyzed the data and wrote the paper.

Acknowledgments: The authors wish to thank Jean-Marc Hardy and Mudasir Ahmed for their technical assistance, and Radu Platon and José Candanedo for their comments during the development of this study. We would like to thank the Office of the Research and Development of Natural Resources Canada for the financial support of this study through the PERD program.

Conflicts of Interest: The authors declare no conflict of interest. The founding sponsors had no role in the design of the study; in the collection, analyses, or interpretation of data; in the writing of the manuscript, and in the decision to publish the results. 


\section{References}

1. Fernandez, N.; Katipamula, S.; Wang, W.; Xie, Y.; Zhao, M.; Corbin, C. D. Impacts of Commercial Building Controls on Energy Savings and Peak Load Reduction; PNNL-25985; Pacific Northwest National Laboratory: Richland, WA, USA, 2017.

2. Li, X.; Wen, J. Review of building energy modeling for control and operation. Renew. Sustain. Energy Rev. 2014, 37, 517-537.

3. Afram, A.; Janabi-Sharifi, F. Theory and application of HVAC control systems-A review of model predictive control (MPC). Build. Environ. 2014, 27, 343-355.

4. Killian, M.; Kozek, M. Ten questions concerning model predictive control for energy efficient buildings. Build. Environ. 2016, 105, 403-412.

5. Candanedo, J.; Saloux, E.; Hardy, J.M.; Platon, R.; RaissiDehkordi, V.; Côté, A. Preliminary assessment of a weather forecast tool for building operation. In Proceedings of the 5th International High Performance Buildings Conference, Purdue, IN, USA, 9-12 July 2018.

6. CanMETEO. Available online: https://www.nrcan.gc.ca/energy/canmeteo/19908 (accessed on 27 August 2019).

7. MATLAB. Available online: https://www.mathworks.com/products/matlab.html (accessed on 18 February 2019).

8. Hammarsten, S. A critical appraisal of energy-signature models. Appl. Energy 1987, 26, 97-110.

(C) 2019 by the authors. Licensee MDPI, Basel, Switzerland. This article is an open access article distributed under the terms and conditions of the Creative Commons Attribution (CC BY) license (http://creativecommons.org/licenses/by/4.0/). 